\title{
Setting the stage for the next step in cardiac amyloidosis imaging: Serial quantitative studies to assess disease activity
}

\author{
Prem Soman, MD, PhD, FRCP (UK), FACC, ${ }^{a}$ and Ahmad Masri, MD $^{\mathrm{a}}$ \\ a Division of Cardiology and the Heart and Vascular Institute, University of Pittsburgh Medical \\ Center, Pittsburgh, PA
}

Received Feb 23, 2017; accepted Feb 24, 2017

doi: 10.1007/s12350-017-0846-7

\section{See related article, pp. 1559-1567}

The term "amyloid" refers to misfolded proteins that tend to aggregate into fibrils, usually with a betapleated structural conformation. ${ }^{1}$ The latter imparts the characteristic apple-green birefringence when stained with Congo-red and viewed under polarized light. The term is attributed to Rudolf Virchow who used it to describe the observed "lardaceous" infiltration of tissues, which was incorrectly thought to be a substance derived from starch (from latin ' amylum',). ${ }^{2}$ Due to already established widespread use, the nomenclature persisted even after the proteinaceous origin of amyloid was subsequently identified. The deposition of amyloid proteins is the basis of several recognized organ-specific and systemic amyloidosis syndromes, neurodegenerative disorders, and prion diseases. ${ }^{3}$

Cardiac amyloid may derived from (1) immunoglobulin light chains produced in excess by a malignant proliferation of plasma cells (AL amyloidosis), or (2) transthyretin ("carrier of thyroxine and retinol', TTR amyloidosis). TTR amyloidosis may be the result of one of several gene mutations $(>100$ identified) manifesting variably as familial neuropathy and/or cardiomyopathy. ${ }^{4}$ It may also occur as a senile form, without a gene mutation. The inherent tendency resident in any protein toward an amyloid state may be

Reprint requests: Prem Soman MD, PhD, FRCP (UK), FACC, Division of Cardiology, and the Heart and Vascular Institute, University of Pittsburgh Medical Center, A-429 Scaife Hall, 200 Lothrop Street, Pittsburgh, PA 15213; premsoman@usa.net

J Nucl Cardiol 2018;25:1571-3.

$1071-3581 / \$ 34.00$

Copyright (C 2017 American Society of Nuclear Cardiology. the basis of the intriguing condition of senile cardiac amyloidosis.

The near coincidence of three factors accounts for a recent focus of interest in cardiac amyloidosis, and the resulting proliferation of literature on the subject. These are as follows: (1) the development of several noninvasive imaging approaches, described below, which have enabled the accurate diagnosis of cardiac amyloidosis without a biopsy. This capability has revealed a much higher prevalence of cardiac amyloidosis than hitherto recognized; (2) an improved understanding of the variable phenotypes associated with the genetic mutations; and (3) the development of several therapeutic agents, currently in pre-clinical and clinical trials, with the promise of improved patient outcome in an area where therapeutic nihilism currently dominates.

The field of cardiac imaging has seen many recent developments in the area of amyloidosis. The rather nonspecific morphological features on two-dimensional echocardiography including biventricular wall thickening with a speckled pattern, enlargement of both atria, thickening of the interventricular septum, and a pericardial effusion, that have long been the tip-off for a referral to cardiac biopsy, have been complemented with a characteristic and more specific apical sparing abnormality of global longitudinal strain. In the inaugural paper describing this finding, its sensitivity and specificity were reported to be $93 \%$ and $82 \%$, respectively, when the echocardiogram showed left ventricular hypertrophy and coronary artery disease was excluded. ${ }^{5}$

Morphological changes analogous to those seen on echocardiography may also be detected by cardiac magnetic resonance (CMR) imaging. CMR offers a superior diagnostic accuracy by virtue of a unique pattern of global subendocardial enhancement of the myocardium, combined with abnormal myocardial and blood-pool gadolinium kinetics (difficulty to null the myocardium, with quick disappearance of gadolinium 
from the blood pool). ${ }^{6}$ The abnormality of global longitudinal strain on echo, and the characteristic findings on CMR, are seen in both AL and TTR amyloidosis.

Perhaps the most consequential development in the noninvasive imaging of cardiac amyloidosis has been the resurgence of Tc-99m pyrophosphate (PYP) imaging as a highly sensitive and specific agent for cardiac ATTR amyloidosis. The diagnostic use of Tc- PYP, a bone imaging agent, for the diagnosis of cardiac amyloidosis is not new, but in the past was confounded by the lack of recognition of its affinity for ATTR, but not AL amyloid. Thus, early studies which included patients with both AL and ATTR amyloidosis reported low diagnostic accuracy. ${ }^{7}$ Recent data from a large, prospective multinational cohort of patients showed exceptionally high diagnostic accuracy when applied specifically to ATTR amyloidosis. ${ }^{8}$ While the specificity for cardiac amyloidosis was near perfect, the specificity for ATTR amyloidosis was slightly lower, reflecting the fact that some patients with AL amyloidosis may show mild uptake of Tc-99m PYP. However, when combined with serum studies which exclude AL amyloidosis, avid uptake of Tc-99m PYP is confirmatory for ATTR amyloidosis with a $100 \%$ specificity, a diagnostic accuracy that is rarely achieved with imaging approaches. $^{8}$

The ability to make a noninvasive diagnosis of cardiac ATTR amyloidosis without a biopsy with a high degree of certainty has resulted in the recognition of a significant prevalence of the disease in defined populations such as heart failure with preserved ejection fraction and severe aortic stenosis with a low transvalvular gradient. ${ }^{9}$ Simultaneously, developments in therapeutics have resulted in a number of agents being currently tested. ${ }^{10}$ These developments, which have the potential to significantly enhance the care of patients with cardiac amyloidosis, have also created new challenges in the evaluation of these patients. Prominent among these challenges is the need to follow disease progression or regression after clinical or clinical trial intervention. It is in this context that the pioneering study by Morgenstern and colleagues should be viewed. ${ }^{11}$

The investigators explored the utility of F-18-labeled sodium fluoride $(\mathrm{NaF})$ for the visual assessment and absolute quantification of tracer uptake in 7 patients with biopsy-proven cardiac amyloidosis ( 3 wild type, 2 mutant, and $2 \mathrm{AL}$ ) compared to 5 controls. The latter were patients with prostate cancer, and had comparable demographics to study patients. Notably, the two patients with mutant ATTR had the V122I mutation, which is unique in its exclusivity to elderly AfricanAmerican male and female patients, and clinical similarity to senile cardiac amyloidosis, albeit with a worse prognosis. ${ }^{4}$ Tracer uptake was evaluated visually and quantitatively using standardized uptake values (SUV) for the entire myocardium (SUVm) and for each of the standard 17 segments. As one considers the study results, it is quite important to keep in mind that this small study is essentially a feasibility pilot. The authors show that with visual assessment and quantitation, F-18 $\mathrm{NaF}$ uptake in ATTR patients was higher (approximately 1.5 times by $\mathrm{SUVm}$ ) than $\mathrm{AL}$ patients or controls. There was no significant difference in uptake between AL patients and controls. Regional SUV calculations showed heterogeneity in myocardial uptake, and with the higher resolution of PET imaging, uptake in atria was discernible in several patients.

While the resurgence of Tc-99m PYP imaging for amyloidosis has greatly simplified the process of obtaining a confirmatory diagnosis of ATTR amyloidosis, prior studies have shown that quantitative serial imaging is not feasible with this approach. ${ }^{12}$ Current attempts to quantify disease activity in cardiac amyloidosis have utilized cardiac biopsy. ${ }^{13}$ However, in addition to the risks associated with its invasiveness, quantification by biopsy may be inaccurate due to the patchy nature of amyloid infiltration and variation in sample size. ${ }^{14}$ Another approach which has been proposed is the use of extra cellular volume (ECV) estimation on CMR. This noninvasive approach lends itself to repeated measures on serial imaging, but may be limited by lack of standardization and incomplete sampling of the myocardium. ${ }^{15,16}$ Thus, there is currently no uniformly accepted and pragmatic method of quantifying the cardiac amyloid load.

So, can PET fulfill this void? The superior spatial resolution compared to SPECT, robust attenuation correction, and superior count statistics make PET potentially a better quantitative instrument than SPECT. The use of SUV as a measure of tracer uptake was introduced to minimize variability resulting from differences in patient size and injected dose. ${ }^{17}$ Nevertheless, experience in oncology has taught us that using the SUV as a quantitative measure of tracer uptake is prone to many influences that introduce measurement variability, which impact its use for serial imaging. ${ }^{18}$ Major influences include partial volume effects, effect of attenuation, biological variability, patient factors such as a change in renal function, and the duration between tracer injection and imaging. ${ }^{17}$ Despite these limitations, current guidelines recommend that SUVs be routinely reported in tumor imaging. ${ }^{19}$

Prior efforts at PET imaging in cardiac amyloidosis used F-18 Florbetapir and C-11 Pittsburgh Compound B (PiB) ${ }^{20,21}$ Quantitative SUV measurements were used to optimize diagnostic sensitivity. However, PET approaches have yet not been studied for the assessment 
of serial changes in amyloid burden. This is an important unmet need, since evidence exists to indicate that therapy which prevents amyloid production can result in resorption of existing deposits, which may then facilitate recovery of organ function. ${ }^{22}$ The paper by Morgenstern and colleagues sets the stage for the next step in radionuclide imaging of cardiac amyloidosis, namely serial quantitative studies. Clearly, many technical challenges will need to be overcome before clinical implementation becomes feasible.

Notably, F-18 Florbetapir and C-11 PiB detect both $\mathrm{AL}$ and ATTR amyloidosis. F-18 NaF, on the other hand, detects only ATTR amyloidosis. The mechanistic basis of the propensity of PET and SPECT bone agents (NaF and Tc-PYP) for ATTR, but not AL amyloidosis, is currently unknown, and may hold an important key to understanding mechanisms of cardiac dysfunction in this intriguing disorder

\section{Disclosures}

Dr. Prem Soman has served on the advisory board of Alnylam Pharmaceuticals. Dr. Masri has no conflicts of interest to report.

\section{References}

1. Toyama BH, Weissman JS. Amyloid structure: Conformational diversity and consequences. Annu Rev Biochem 2011;80:557-85.

2. Kyle RA. Amyloidosis: A convoluted story. Br J Haematol 2001;114:529-38.

3. Pulawski W, Ghoshdastider U, Andrisano V, Filipek S. Ubiquitous amyloids. Appl Biochem Biotechnol 2012;166:1626-43.

4. Ruberg FL, Berk JL. Transthyretin (TTR) cardiac amyloidosis. Circulation 2012;126:1286-300.

5. Phelan D, Collier P, Thavendiranathan $\mathrm{P}$, et al. Relative apical sparing of longitudinal strain using two-dimensional speckletracking echocardiography is both sensitive and specific for the diagnosis of cardiac amyloidosis. Heart 2012;98:1442-8.

6. Maceira AM, Joshi J, Prasad SK, et al. Cardiovascular magnetic resonance in cardiac amyloidosis. Circulation 2005;111:186-93.

7. Gertz MA, Brown ML, Hauser MF, Kyle RA. Utility of technetium Tc $99 \mathrm{~m}$ pyrophosphate bone scanning in cardiac amyloidosis. Arch Intern Med 1987;147:1039-44.
8. Gillmore JD, Maurer MS, Falk RH, Merlini G, Damy T, Dispenzieri A, et al. Nonbiopsy diagnosis of cardiac transthyretin amyloidosis. Circulation 2016;133(24):2404-12.

9. González-López E, Gallego-Delgado M, Guzzo-Merello G, et al. Wild-type transthyretin amyloidosis as a cause of heart failure with preserved ejection fraction. Eur Heart J 2015;36:2585-94.

10. Castaño A, Bokhari S, Maurer MS. Unveiling wild-type transthyretin cardiac amyloidosis as a significant and potentially modifiable cause of heart failure with preserved ejection fraction. Eur Heart J 2015;36:2595-7.

11. Morgenstern R, Yeh R, Castano A, Maurer MS, Bokhari S. ${ }^{18}$ Fluorine sodium fluoride positron emission tomography, a potential biomarker of transthyretin cardiac amyloidosis. J Nucl Cardiol. 2017. doi:10.1007/s12350-017-0799-x.

12. Castano A, DeLuca A, Weinberg R, et al. Serial scanning with technetium pyrophosphate (99mTc-PYP) in advanced ATTR cardiac amyloidosis. J Nucl Cardiol 2016;23:1355-63.

13. Kristen AV, Brokbals E, Aus dem Siepen F, et al. Cardiac amyloid load: A prognostic and predictive biomarker in patients with lightchain amyloidosis. J Am Coll Cardiol 2016;68:13-24.

14. Ruberg FL, Fontana M, Gillmore JD. Should histologic determination of amyloid load determine management decisions in lightchain amyloidosis? J Am Coll Cardiol 2016;68:2493-4.

15. Fontana M, Pica S, Reant P, et al. Prognostic value of late gadolinium enhancement cardiovascular magnetic resonance in cardiac amyloidosis. Circulation 2015;132:1570-9.

16. Kristen AV, Brokbals E, Aus dem Siepen F, et al. Reply: Should histologic determination of amyloid load determine management decisions in light-chain amyloidosis? J Am Coll Cardiol 2016;68:2494-5.

17. Kinahan PE, Fletcher JW. PET/CT standardized uptake values (SUVs) in clinical practice and assessing response to therapy. Semin Ultrasound CT MR 2010;31:496-505.

18. Boellaard R, Krak NC, Hoekstra OS, Lammertsma AA. Effects of noise, image resolution, and ROI definition on the accuracy of standard uptake values: a simulation study. J Nucl Med 2004;45:1519-27.

19. Delbeke D, Coleman RE, Guiberteau MJ, et al. Procedure guideline for tumor imaging with 18F-FDG PET/CT 1.0. J Nucl Med 2006;47:885-95.

20. Dorbala S, Vangala D, Semer J, et al. Imaging cardiac amyloidosis: a pilot study using (1)(8)F-florbetapir positron emission tomography. Eur J Nucl Med Mol Imaging 2014;41:1652-62.

21. Lee SP, Lee ES, Choi H, et al. 11C-Pittsburgh B PET imaging in cardiac amyloidosis. JACC Cardiovasc Imaging 2015;8:50-9.

22. Merlini G, Bellotti V. Molecular mechanisms of amyloidosis. N Engl J Med 2003;349:583-96. 